\title{
Design and Performance of a High Temperature Superconducting Axial Flux Generator
}

\author{
Trapanese M. ${ }^{1}$, Member, IEEE, Franzitta V. ${ }^{1}$, Viola A. ${ }^{1}$ \\ ${ }^{1}$ DIEETCAM University of Palermo, Palermo 90128 Italy
}

In this paper, a high temperature axial flux (HTSAF) generator is presented. In this generator the excitation of the generator is obtained by using some high temperature superconducting magnets. In order to reduce the negative effects of vibrations, the excitation is located on the stationary part of the generator. Starting, running and endurance tests of the machine are presented.

Index Terms-Superconducting materials, magnetic materials, electric machines, power systems.

\section{INTRODUCTION}

A $\mathrm{N}$ IMPORTANT quality index of electric machine is the power density: it is known that axial flux machines tend to have a higher power density than the radial machines. As a result, they are used more and more in applications where the power density is a critical issue: renewable energy production[1], automotive etc. However, the best way to enhance the power density is to increase the value of the airgap field, and this could be achieved by using a superconducting excitation. Many projects have been developed in the world in order to develop both a superconducting generator and a superconducting motor, but in most of these programs the geometry has been based on radial generators [2-5]. All of these approaches show brilliantly the feasibility of a superconducting generator, but some drawbacks are eventually linked to the fact that the low temperature area must be guaranteed in the moving part of the generator. In this paper, a high temperature axial flux (HTSAF) generator is presented. In this generator the excitation is obtained by using some high temperature superconducting magnets which are able to trap induction field quasi-permanently. The magnets are installed in the stationary part of the generator inside a thermal vessel which contains the coolant to reduce the thermal losses. This vessel is linked to the cooling system through two ducts. Windings are located on the rotating part of the generator and are linked to the power electronics section of the generator trough a slip and ring system. In the paper, the main features of high temperature superconducting magnets are presented in section II, in section III the characteristics of the generator are reported and in section IV the experimental results are presented. Finally, in section V conclusions are drawn.

\section{High TEMPERATURE SUPERCONDUCTING MAGNETS}

High temperature superconducting permanent magnets (HTSPM) are pieces of high temperature superconducting material which are able to trap induction field, this ability is a consequence of the pinning phenomenon of magnetic lines which occurs in high temperature superconductors. In order to be able to trap magnetic field, these magnets must be cooled in a magnetic field (that will be called activation field in the following part of the paper) and successively the external field can be removed leaving a field trapped inside the HTSPM. The value of trapped field depends on several parameters such as the cooling temperature, the value of the activation field, the dimensions of magnets, the fabrication method of the materials etc. Because of the fact that the trapping phenomenon is related to the pinning of magnetic lines, the distribution of the field on the surface of the HTSPM is different from that of a permanent magnet. Moreover, the value of the trapped field decreases with time at a rate of about $5 \%$ per week. This rate depends strongly on the mechanical disturbances that the magnets face. The magnet used in this paper is made of $\mathrm{YBa} 2 \mathrm{Cu} 3 \mathrm{O} 7$.

\section{GENERATOR}

As already said in the previous section, mechanical and thermal disturbances can seriously reduce the induction field trapped in a HTSPM. As a result in this paper we propose a design which can solve this problem. In fig. 1 this design is shown. A reliable numerical magnetic analysis of this generator is quite difficult because the field distribution on the surface of HTSPM cannot be modeled mathematically through a simple approach fully integrable in a FEM based design tool. However, in order to obtain a preliminary design of the generator, the design of the generator has been done by a commercial FEM by assuming that the field of a HTSPM can be modeled in the same way of the field of a PM. This approach allows to obtain a more rapid design.

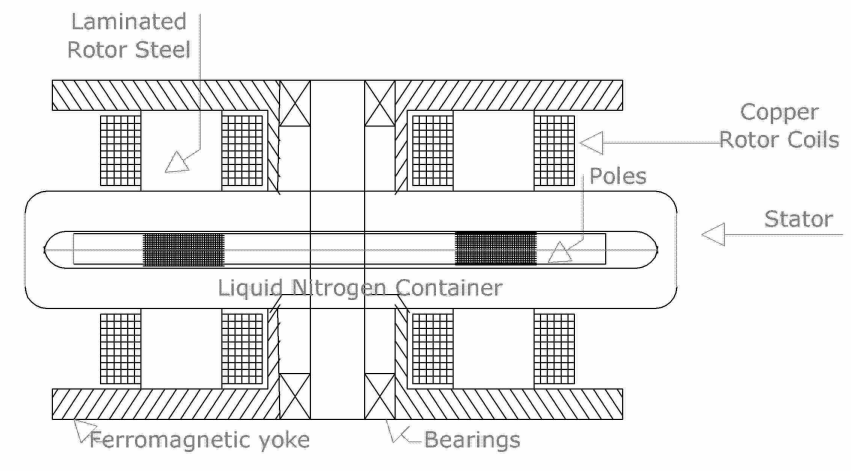

Fig.1 Cross section of the generator 
In this generator the excitation is obtained by using some high temperature superconducting magnets (poles in Fig.1). The magnets are installed in the stationary part of the generator inside a thermal vessel which contains the coolant and reduces the thermal losses. This vessel is linked to the cooling system through two ducts. Windings are located on the rotating part of the generator and are linked to the power electronics section of the generator through a slip and ring system. Due to the fact that this generator has been built in order to work at a maximum field of $2 \mathrm{~T}$, a ferromagnetic yoke has been installed, however, in a new version of the generator, which works at $4 \mathrm{~T}$, this part of the generator has not been included in the design. The winding coils are connected to a slip-ring in order to collect the electromotive force.

The structure of the stator is equal to the one of the rotor in [5]. It consists of a disk containing 8 HTSPMs. Each HTSPM has a $2 \mathrm{~cm}$ diameter and is $1 \mathrm{~cm}$ thick.

The rotor has a double sided structure. This structure reduces the vertical forces between the stator and the rotor. The windings are made of copper. The rotor runs at room temperature. The pole shoes and the yoke are made of laminated steel.

\section{EXPERIMENTAL RESULTS}

Several tests have been done both on the HTSPM as well on the HTSAF generator.

\section{A. Tests of HTSPM.}

As well as HTSPM tests are concerned, two tests have been performed: one has measured the maximum trapped field vs. activation field, the other measured the loss of field vs. time.

In fig. 2 the maximum trapped field dependence on activation field is reported. In this test, temperature was measured by a PT100 sensor located inside the vessel and the magnetic field was measured through hall sensors located outside the vessel. In this text, the generator is not rotating.

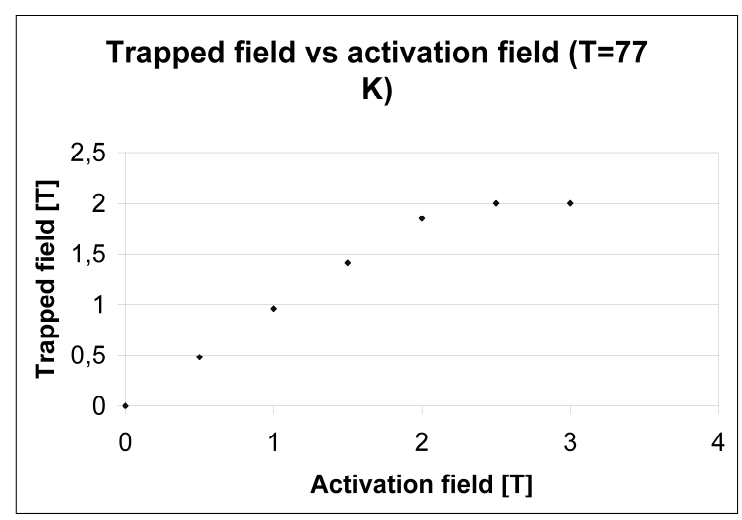

Fig. 2. Trapped field vs activation field

From fig. 2 it can be seen that at a temperature of $77 \mathrm{~K}$ there is a saturation value of the trapped field equal to $2 \mathrm{~T}$. In order to reach higher level of trapped field, lower temperature are needed

In fig. 3 the loss of field variation (at stand still) with time is reported. In this test, temperature was measured by a PT100 sensor located inside the vessel, magnetic field was measured through hall sensors located outside the vessel, and time by a computer clock.

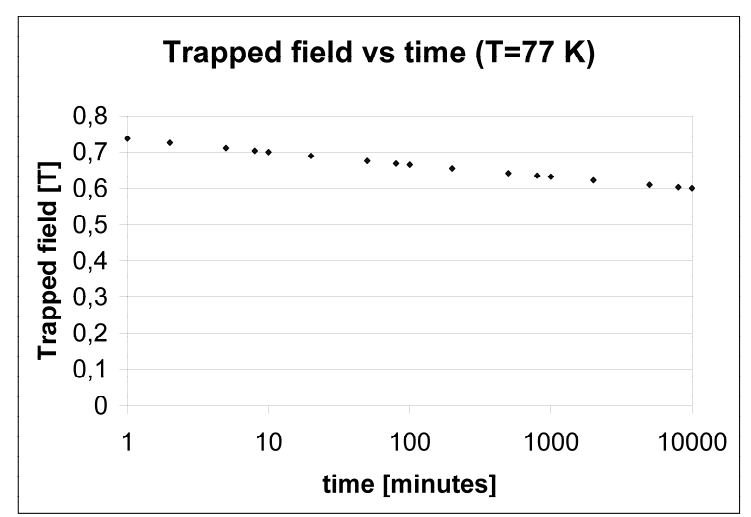

Fig .3. The loss of field with time. In this test, the generator is not rotating.

The loss field variation can be greatly accelerated by the presence of vibration. Fig. 3 shows clearly that in order to guarantee a reliable operation of the generator, it must have the capability to re-magnetized the magnets. As a result, a complete design of the generator must include the possibility to provide the re-activation. The current design can deal with this issue partially, and some preliminary tests on the possibility to deal with this problem are reported in the subsection B in the endurance test. The effect shown in fig.3 influences strongly the output voltage of the system, as a result, in order to define the output voltage of the generator we have decided to wait a settling time of the generator equal to 10 minutes. The output voltages reported in tab. 1 are obtained accordingly to this definition.

\section{B. Generator Tests}

In order to characterize the generator the following tests have been done:
1) start test;
2) running test;
3) endurance test.

1) Start Test.

The objective of the start test is to verify the possibility of magnetizing the magnets placed inside the generator. The starting procedure can be divided in three phase: cooling down of the magnets, magnetization and verification of the level of the trapped field. Temperature was measured by using a PT 100 sensor placed inside the vessel and magnetic field by using a Hall sensor placed in the air gap. 
The cooling is performed by injecting liquid nitrogen inside the vessel.

In order to magnetize the magnets inside the generator, a pulsed current has been injected in the windings.

The maximum air gap field which has been reached is $1.8 \mathrm{~T}$ which allowed $0.7 \mathrm{~T}$. It was not possible to inject higher current because of the thermal limitation on the copper winding. As a result, if the machine has to guarantee a higher activation field, a dedicated design of the windings is needed

\section{2) Running Test}

The objective of the running test is to measure the emf collected on the slip-ring system. In table 1 the open circuit voltage is reported for various revolving speeds.

Speed was measured by a digital rotating encoder, voltage through an ordinary voltmeter and the generator was coupled to a driving motor

The generator has not yet been loaded because a new experimental setup is needed in order to study the armature reaction effect on the HTSPM.

Table I Open circuit voltage

\begin{tabular}{|c|c|}
\hline Speed [rpm] & Voltage (line to line) \\
\hline 200 & 10.1 \\
\hline 400 & 20.4 \\
\hline 600 & 30.5 \\
\hline 1000 & 45.6 \\
\hline 1200 & 53.4 \\
\hline 1500 & 64.2 \\
\hline
\end{tabular}

Table I shows how at higher speed the linearity between Voltage and speed is lost. This is caused by the fact that the higher the speed, the higher the mechanical disturbances that reduce the trapped field and consequently the open voltage.

The voltages reported in tab. 1, have been collected, after a settling time of the generator equal to 10 minutes, as said above.

\section{3) Endurance Test.}

The objective of the endurance test is to verify the persistence of the trapped flux in the magnets. The test has been performed by measuring the open circuit voltage as a function of time at constant speed. Three speed values have been experimentally verified $(1200 \mathrm{rpm}, 400 \mathrm{rpm}$ and 200 rpm)and in each case the trapped field was equal to 0.7 Tesla. Fig .4 shows how the voltage has a high value in the inistial phase of the test and reaches a plateau after 15 minutes at $1200 \mathrm{rpm}$. At $400 \mathrm{rpm}$ there is the same effect but the settling time is faster but the reduction of the voltage is less evident. At $200 \mathrm{rpm}$ this effect is negligible and the voltage seems to be almost constant. The reproducibility of this tests was on the order of $10 \%$. Also in this case, voltage was measured by an ordinary voltmeter, time by a computer clock and the generator was coupled to a prime motor. The authors believe that this effect was caused by the vibration of the machine: the higher the speed, the higher the vibration and therefore the faster the reduction of the superconductivity of the magnets. At a low level of vibration, this effect seems to be negligible.

Some endurance tests in loaded conditions have been done. In these conditions, magnets were continuously energized by a suitable electronic control of the output current in order to compensate the a.c. losses of the magnets and the decaying of the voltage was not observed.

\section{Output Voltage vs time $(\mathrm{T}=77 \mathrm{~K})$}

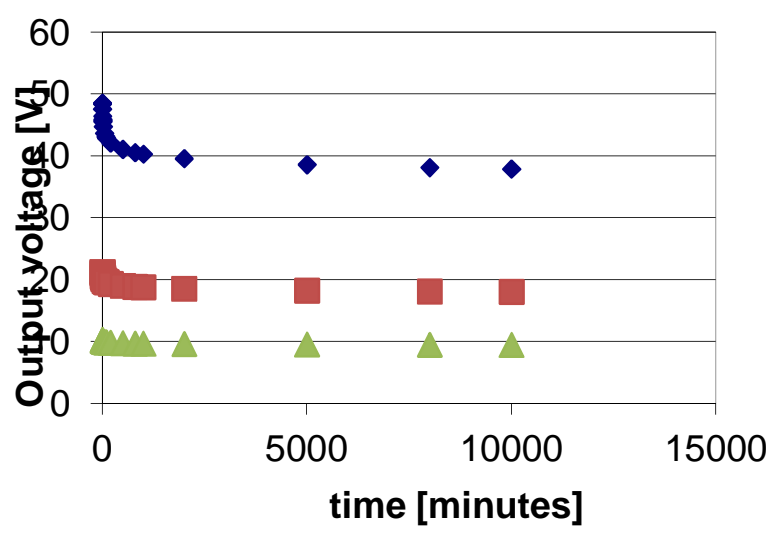

Fig.4 Endurance test. Output voltage vs time. Diamonds indicate a rotating speed equal to $1200 \mathrm{rpm}$ squares a speed equal to $400 \mathrm{rpm}$, triangles a speed equal to $200 \mathrm{rpm}$.

\section{CONCLUSIONS}

In this paper, a high temperature axial flux (HTSAF) generator is presented. In this generator the excitation of the generator is obtained by using some high temperature superconducting magnets. The magnets are installed in the stationary part of the generator inside a thermal vessel which contains the coolant and reduces the thermal losses. This vessel is linked to the cooling system through two ducts. The winding is located on the rotating part of the generator and are linked to the power electronics section of the generator through a slip and ring system. The start test, the running test and the endurance test have been performed on the generator. It has been experimentally shown that generator can be magnetized at a level that allows the generation of an electromotive force and that this level of magnetization can be kept for a time span no shorter than hours.

\section{ACKNOWLEDGMENT}

This work was supported by Ministero dell'Ambiente of Republic of Italy under IMPETUS project. 


\section{REFERENCES}

[1] V Di Dio, V Franzitta, F Muzio, G Scaccianoce, M Trapanese, " The use of sea waves for generation of electrical energy and Hydrogen" OCEANS 2009, MTS/IEEE Biloxi-Marine, 2009.

[2] Parker, J., Towne, R., "Superconducting AC generators", IEEE Transactions on Magnetics, Volume: 12 , Issue: 6, 1976, pp 909-914.

[3] Singh, S.S., Scherbarth, D.W, Ortoli, E.S., Repp, J.R., Christianson O.R., Parker, J.H. and Carr, J.W., "Conceptual Design of a High Temperature Superconducting Generator", IEEE Transactions on Applied Superconductivity, Volume: 9, Issue: 2, 1999, pp 1237-1240.
[4] Al-Mosawi M.K., Beduz, C. and Yang Y., "Construction of a 100 kVA high temperature superconducting synchronous generator", IEEE Transactions on Applied Superconductivity, Volume: 15, Issue: 2, 2005, pp 2182-2185.

[5] Weinstein, R and Sawh, R., "An experimental generator using high temperature superconducting quasi permanent magnets", IEEE Transactions on Applied Superconductivity, Volume: 5, Issue: 2, 1995, pp $441-444$.

Manuscript received Novemebr, 6, 2012 (date on which paper was submitted for review). Corresponding author: M.Trapanese (e-mail: marco.trapanese@unipa.it phone: +39-0916615292; optional fax: +39091488452) 